\title{
Excitation of normal modes of a thin elastic plate by moving dislocations
}

\author{
Rodrigo ARIAS ANd FERnANdo Lund \\ Departamento de Física, Facultad de Ciencias Físicas y Matemáticas \\ Universidad de Chile, Casilla 487-3, Santiago, Chile
}

\begin{abstract}
We study the excitation of harmonic waves in thin elastic samples by a single dislocation in arbitrary motion. We consider both screw and edge dislocations that move perpendicularly to the surfaces of the layer. In Fourier space the displacement velocity and dynamic stress fields generated by the motion of the dislocations are factored as the product of two terms: one depends on the motion of the dislocation only, while the other is independent of it, and represents the medium's response. The latter term exhibits poles at frequencies that satisfy the dispersion relation of the harmonic modes of the plate. In the case of a screw dislocation the modes that are excited are a subfamily of the antisymmetric Rayleigh-Lamb modes. For an edge dislocation a subfamily of the symmetric Rayleigh-Lamb modes is excited, as well as the lowest lying shear mode. The expression corresponding to a uniformly moving screw is worked out in detail; it has singular behavior at velocities coincident with the phase velocities of the allowed modes.
\end{abstract}

\section{Introduction}

Recent experimental results [1, 2, 3, 目, 5, 6] in fast crack propagation in brittle materials, such as glass and plexiglass, have provided evidence that crack propagation is dynamically unstable, and bounds that separate different crack behavior have been identified experimentally in terms of the crack tip velocity. For example, when the crack tip velocity surpasses $0.5 c_{R}$, with $c_{R}$ the Rayleigh wave velocity, there are strong sound emissions, oscillations of the crack tip velocity, and the surfaces left by the crack become quite rough.

A scenario for a dynamic instability that could explain in a unified way the experimentally observed features of acoustic emissions, crack tip velocity oscillations and surface roughness has been recently proposed by one of us [7]. This scenario is based on continuum elasticity, and it incorporates the fact that a crack in motion can be modeled as a continuous, time dependent, distribution of dislocations. These moving dislocations, when propagating in a medium that is itself oscillating, are subjet to two forces: one is the usual Peach-Koehler force, and the second a force that does no work [8], in this sense analogous to the Lorentz magnetic force acting on a moving charged particle. This latter force that does no work is proportional to the dislocation's velocity, perpendicular to it, and it is also proportional to the time derivative of the particle displacement generated by time dependent external loads. The dynamic instability would occur by the following mechanism: 1) the crack begins to propagate and starts emitting elastic radiation; 2) normal modes of vibration of the plate are established, and they are responsible for a new oscillating force that 
does no work and that is proportional to the crack tip velocity; 3) the crack responds to this new periodic forcing by emitting radiation mainly at the same frequencies at which it is being forced. This radiation reinforces the normal modes, that in turn act on the crack tip: this is the instability.

The samples of brittle material that are used in experiments [1, 2, 3, 4, 5, 6] are typically a few $\mathrm{mm}$ thick, and $\sim 20 \mathrm{~cm}$ in width and length. Thus, it appears that useful insights into the underlying physics may be obtained by considering the samples as thin layers; that is, elastic layers of finite thickness and infinite in-plane dimensions. In this case the elastic sample is a wave guide whose possible horizontally polarized shear modes, and its mixed character Rayleigh-Lamb modes are well known [9]. In this work we have investigated the excitation of harmonic waves in such a wave guide by a dislocation, perpendicular to the surfaces of the layer, in arbitrary motion. This represents a first step towards a firm analytic foundation for the scenario proposed in the previous paragraph. The principal results of this work are expressions, in Fourier space, that describe the displacement velocity field and the stresses due to screw or edge dislocations moving perpendicularly to the surfaces of the plate with arbitrary velocity, and the identification of the specific wave modes excited in each case. The relevant expressions are given as convolutions of a source function localized at the dislocation position with the medium's response, of which the Rayleigh-Lamb modes are poles.

In order to proceed, we use a formulation reported elsewhere 11 for the stress and velocity generated by a moving dislocation in finite elastic samples. This formulation is a generalization of line integral representations first found by Mura [10] in the case of an infinite medium. The specialization of these results for a thin plate is given in Section 2. Section 3 contains the determination of amplitudes, whose precise definition is given in the text, associated with each possible harmonic mode. Expressions for particle velocity in terms of those amplitudes and a source function associated with the moving dislocation are given in Section 4 for a screw and in Section 5 for an edge dislocation. Section 6 discusses the corresponding stresses. Section 7 carries these results further in the special case of a uniformly moving dislocation, with the well known result of a dislocation in uniform motion in an infinite medium carried along for comparison. A discussion of the implications of these results for our current understanding of the brittle fracture of thin

plates is offered in Section 8, and Section 9 has concluding remarks. The Fourier components of the Green's function of a thin plate [12] are written down explicitely in an Appendix.

\section{Displacement velocity due to a dislocation loop moving in a finite sample}

We consider small displacements $U_{m}(\vec{x}, t)$ in a homogeneous elastic medium of density $\rho$ and elastic constants $C_{i j k l}$ [9]. A general formula for the velocity and stress fields generated by a dislocation loop undergoing arbitrary motion in an infinite medium was obtained some years ago by Mura [10]. It is written as a convolution of the medium's impulse response with a source localized along the dislocation loop (i.e. independent of the slip plane). These formulae have been generalized to the case of a bounded elastic medium, as well as the formula for the displacement field that involves integration over succesive slip planes [11]. The latter expression for the displacement field is:

$$
U_{m}(x, t)=-b_{i} \int_{-\infty}^{\infty} d t^{\prime} \int_{S\left(t^{\prime}\right)} d S_{j}^{\prime} \sigma_{i j}^{G m}\left(x^{\prime}, x ; t-t^{\prime}\right),
$$


(attention should be paid to the order of the variables) where $\sigma_{i j}^{G m}\left(x, x^{\prime} ; t-t^{\prime}\right)$ is the elastic stress associated with the Green's function:

$$
\sigma_{i j}^{G m}\left(x, x^{\prime} ; t-t^{\prime}\right) \equiv C_{i j k l} \frac{\partial}{\partial x_{k}} G_{l m}\left(x, x^{\prime} ; t-t^{\prime}\right) .
$$

Eq. (11) is the analog of Mura's relation [10]. Since the problem is homogeneous under time translations it is possible to obtain an expression for particle velocity, the time derivative of particle displacement $U_{m}$ that involves an integral along the dislocation loop only. Indeed, since $\partial G_{m k} / \partial t=$ $-\partial G_{m k} / \partial t^{\prime}$, the displacement velocity becomes:

$$
\frac{\partial U_{m}}{\partial t}(x, t)=b_{i} \int_{-\infty}^{\infty} d t^{\prime}\left\{\frac{\partial}{\partial t^{\prime}}\left[\int_{S\left(t^{\prime}\right)} d S_{j}^{\prime} \sigma_{i j}^{G m}\left(x^{\prime}, x ; t-t^{\prime}\right)\right]-\int_{\frac{d S}{d t^{\prime}}\left(t^{\prime}\right)} d S_{j}^{\prime} \sigma_{i j}^{G m}\left(x^{\prime}, x ; t-t^{\prime}\right)\right\} .
$$

The first term is zero since the Green's function vanishes at $t-t^{\prime}= \pm \infty$. Also, since $\int_{d S\left(t^{\prime}\right) / d t^{\prime}} d S_{j}^{\prime}=$ $\epsilon_{j p q} \int_{L\left(t^{\prime}\right)} d l_{q}^{\prime} V_{p}\left(x^{\prime}, t^{\prime}\right)$, where $L\left(t^{\prime}\right)$ is the dislocation loop bounding the slip plane $S\left(t^{\prime}\right), V_{p}\left(x^{\prime}, t^{\prime}\right)$ is the loop's local velocity and $\epsilon_{j p q}$ the completely antisymmetric tensor in three dimensions, we have:

$$
\frac{\partial U_{m}}{\partial t}(x, t)=-b_{i} \int_{-\infty}^{\infty} d t^{\prime} \int_{L\left(t^{\prime}\right)} d l_{q}^{\prime} \sigma_{i j}^{G m}\left(x^{\prime}, x ; t-t^{\prime}\right) \epsilon_{j p q} V_{p}\left(x^{\prime}, t^{\prime}\right) .
$$

This expression for the particle velocity generated by a single dislocation loop within a finite elastic sample can also be obtained as a special case of the formulation of Chishko [12 for the particle velocity generated by a continuous distribution of dislocations in a thin elastic plate.

We now specialize to the case of an infinite plate of thickness $2 h$, with plane boundaries located at $z= \pm h$. The appropriate Green's function was calculated by Chishko [12] and it has the form:

$$
G_{i m}\left(z, z^{\prime} ; \vec{R}-\vec{R}^{\prime} ; t-t^{\prime}\right)=G_{i m}^{\infty}\left(\vec{x}-\vec{x}^{\prime} ; t-t^{\prime}\right)-H_{i m}\left(z, z^{\prime} ; \vec{R}-\vec{R}^{\prime} ; t-t^{\prime}\right),
$$

with $G_{i m}^{\infty}$ the Green's function appropriate for an infinite isotropic medium, and $H_{i m}$ a kernel that cancels the normal stresses due to $G_{i m}^{\infty}$ at the free surfaces. $H_{i m}$ is the following convolution:

$$
\begin{aligned}
H_{i m}\left(z, z^{\prime} ; \vec{R}-\vec{R}^{\prime} ; t-t^{\prime}\right)= & \Theta\left(t-t^{\prime}\right) \sum_{a=1,2} \int_{0}^{\left(t-t^{\prime}\right)} d \tau \int d S_{a}^{\prime \prime} \\
& \times G_{i n}^{P}\left(z, \xi^{a} ; \vec{R}-\vec{R}^{\prime \prime} ; t-t^{\prime}-\tau\right) \sigma_{n z}^{\infty m}\left(\xi^{a}-z^{\prime} ; \vec{R}^{\prime \prime}-\vec{R}^{\prime} ; \tau\right),
\end{aligned}
$$

with $d S_{a}^{\prime \prime}= \pm d x d y$ for $a=1,2$ or $z^{\prime \prime}= \pm h$; $\Theta$ is the step function, and $\xi^{a}= \pm h$. Here $\sigma_{n z}^{\infty m}$ is the elastic stress associated with the infinite medium Green's function, and $G_{i n}^{P}\left(z, \xi^{a} ; \vec{R}-\vec{R}^{\prime} ; t-t^{\prime}\right)$ is the Green's function of the homogeneous boundary value problem of the plate: it corresponds to the displacement in direction $i$ produced by a point force applied at the surface in direction $n$ at position $\left(\xi^{a}, \vec{R}^{\prime}\right)$, and time $t^{\prime}$ :

$$
\rho \frac{\partial^{2}}{\partial t^{2}} G_{i n}^{P}\left(z, \xi^{a} ; \vec{R}-\vec{R}^{\prime} ; t-t^{\prime}\right)-C_{i j l m} \frac{\partial^{2}}{\partial x^{j} \partial x^{l}} G_{m n}^{P}\left(z, \xi^{a} ; \vec{R}-\vec{R}^{\prime} ; t-t^{\prime}\right)=0,
$$

with $\xi^{a}= \pm h$, and subject to the following boundary conditions at the surfaces:

$$
\begin{aligned}
\sigma_{i z}^{P n}\left(\xi^{b}, \xi^{a} ; \vec{R}-\vec{R}^{\prime} ; t-t^{\prime}\right) & =C_{i z l m} \frac{\partial}{\partial x^{l}} G_{m n}^{P}\left(\xi^{b}, \xi^{a} ; \vec{R}-\vec{R}^{\prime} ; t-t^{\prime}\right) \\
& = \pm \delta^{a b} \delta_{i n} \delta\left(\vec{R}-\vec{R}^{\prime}\right) \delta\left(t-t^{\prime}\right),
\end{aligned}
$$


where \pm corresponds to $a=1,2$ or to the surfaces $z^{\prime}=\xi^{a}= \pm h$ respectively.

For convenience, we have reproduced the explicit expressions for the Fourier transforms of $G_{m n}^{P}\left(z, \xi ; \vec{R}-\vec{R}^{\prime} ; t-t^{\prime}\right), g_{m n}^{P \omega}(z, \xi \mid \vec{k})$, from Ref. 112] in an Appendix:

$$
g_{m n}^{P \omega}(z, \xi \mid \vec{k}) \equiv \int_{-\infty}^{\infty} d t \int d \vec{R} e^{-i \omega t+i \vec{k} \cdot \vec{R}} G_{m n}^{P}(z, \xi ; \vec{R} ; t) .
$$

\section{Harmonic modes of a thin plate}

Harmonic modes in a thin elastic layer have properties, such as polarization and dispersion relations, that are well known [9]. In particular, the dispersion relation can be read off from the poles of the Fourier transform of the Green's function $G_{i j}^{P}, g_{i j}^{P \omega}(z \mid \vec{k})$ (see Eqs. (57)- 60 ) in the Appendix). Poles of $g_{i j}^{P \omega}(z \mid \vec{k})$ occur when $\Delta_{a}\left(k^{2}, \omega^{2}\right)=0, \Delta_{s}\left(k^{2}, \omega^{2}\right)=0$ and when $\sinh \left(q_{t} h\right)=0$, $\cosh \left(q_{t} h\right)=0$, with $q_{l, t} \equiv \sqrt{k^{2}-\omega^{2} / c_{l, t}^{2}}$, and $c_{l}$ (resp. $c_{t}$ ) are the speed of longitudinal (resp. transverse) waves in bulk material.

The poles $\Delta_{a}\left(k^{2}, \omega^{2}\right)=0$ of $g_{i j}^{P \omega}(z, \xi \mid \vec{k})$ give rise to antisymmetric modes, and we now determine their amplitudes, which will be needed in the next section, by taking the limit:

$$
\left(v_{A}^{j}\right)_{i}=\lim _{\Delta_{a} \rightarrow 0} \Delta_{a} g_{i j}^{P \omega}(z, \xi \mid \vec{k}) .
$$

In this way three linearly independent modes, labelled by " $j$ " are obtained. They are vectors whose components are labelled by " $i$ ". These antisymmetric modes, of mixed character, are for $j=\beta=1,2=x, y$ and $j=3=z$ respectively:

$$
\begin{aligned}
& \vec{v}_{A}^{\beta}(z \mid \vec{k})=i k_{\beta}\left[\left(k^{2}+q_{t}^{2}\right) \sinh \left(q_{l} h\right) \cosh \left(q_{t} z\right)-2 q_{l} q_{t} \sinh \left(q_{t} h\right) \cosh \left(q_{l} z\right)\right] \hat{z} \\
& +\left\{\frac{k_{\alpha} k_{\beta}}{k^{2}} q_{t}\left[\left(k^{2}+q_{t}^{2}\right) \sinh \left(q_{l} h\right) \sinh \left(q_{t} z\right)-2 k^{2} \sinh \left(q_{t} h\right) \sinh \left(q_{l} z\right)\right]\right. \\
& +\left(\delta_{\alpha \beta}-\frac{k_{\alpha} k_{\beta}}{k^{2}}\right)\left[\frac{\left(k^{2}+q_{t}^{2}\right)^{2}}{q_{t}} \sinh \left(q_{l} h\right) \sinh \left(q_{t} z\right)\right. \\
& \left.\left.-4 q_{l} k^{2} \tanh \left(q_{t} h\right) \cosh \left(q_{l} h\right) \sinh \left(q_{t} z\right)\right]\right\} \hat{e}_{\alpha} \\
& \vec{v}_{A}^{z}(z \mid \vec{k})=q_{l}\left[\left(k^{2}+q_{t}^{2}\right) \cosh \left(q_{t} h\right) \cosh \left(q_{l} z\right)-2 k^{2} \cosh \left(q_{l} h\right) \cosh \left(q_{t} z\right)\right] \hat{z} \\
& -i k_{\alpha}\left[\left(k^{2}+q_{t}^{2}\right) \cosh \left(q_{t} h\right) \sinh \left(q_{l} z\right)-2 q_{l} q_{t} \cosh \left(q_{l} h\right) \sinh \left(q_{t} z\right)\right] \hat{e}_{\alpha} .
\end{aligned}
$$

A mode that is not linearly independent of the latter can be obtained as: $\vec{v}_{A}^{L}(z \mid \vec{k}) \equiv-k_{\beta} \vec{v}_{A}^{\beta}(z \mid \vec{k})$. It will be seen below that this is the mode excited by a moving screw dislocation:

$$
\begin{aligned}
\vec{v}_{A}^{L}(z \mid \vec{k}) & =-i k^{2}\left[\left(k^{2}+q_{t}^{2}\right) \sinh \left(q_{l} h\right) \cosh \left(q_{t} z\right)-2 q_{l} q_{t} \sinh \left(q_{t} h\right) \cosh \left(q_{l} z\right)\right] \hat{z} \\
& +q_{t} k_{\alpha} \hat{e}_{\alpha}\left[2 k^{2} \sinh \left(q_{t} h\right) \sinh \left(q_{l} z\right)-\left(k^{2}+q_{t}^{2}\right) \sinh \left(q_{l} h\right) \sinh \left(q_{t} z\right)\right] .
\end{aligned}
$$

In an analogous way, the amplitude of the symmetric modes corresponding to $\Delta_{s}\left(k^{2}, \omega^{2}\right)=0$ are obtained as:

$$
\left(v_{S}^{j}\right)_{i} \sim \lim _{\Delta_{s} \rightarrow 0} \Delta_{s} g_{i j}^{P \omega}(z, \xi \mid \vec{k}) .
$$


It will also be seen below that the mode excited by a moving edge dislocation corresponds to $j=3=z$ :

$$
\begin{aligned}
\vec{v}_{S}^{z}(z \mid \vec{k}) & =q_{l}\left[\left(k^{2}+q_{t}^{2}\right) \sinh \left(q_{t} h\right) \sinh \left(q_{l} z\right)-2 k^{2} \sinh \left(q_{l} h\right) \sinh \left(q_{t} z\right)\right] \hat{z} \\
& -i k_{\alpha}\left[\left(k^{2}+q_{t}^{2}\right) \sinh \left(q_{t} h\right) \cosh \left(q_{l} z\right)-2 q_{l} q_{t} \sinh \left(q_{l} h\right) \cosh \left(q_{t} z\right)\right] \hat{e}_{\alpha} .
\end{aligned}
$$

The shear normal modes are of transverse character and polarized parallel to the surfaces of the plate. The symmetric shear modes can be obtained by multiplying $g_{i j}^{P}$ by $\sinh \left(q_{t} h\right)$ and taking the limit $\sinh \left(q_{t} h\right)=0$, or $q_{t} h=i n \pi, n=1,2, \ldots$. Thus:

$$
\vec{v}_{S}^{\beta}(z \mid \vec{k})=k^{2}\left(\delta_{\alpha \beta}-\frac{k_{\alpha} k_{\beta}}{k^{2}}\right) \cos \left(\frac{n \pi}{h} z\right) \hat{e}_{\alpha} .
$$

The antisymmetric shear modes correspond to multiplying $g_{i j}^{P}(z, \xi \mid \vec{k})$ by $\cosh \left(q_{t} h\right)$, and then taking the limit $\cosh \left(q_{t} h\right)=0$, or $q_{t} h=i(2 n+1) \pi / 2, n=1,2, \ldots$ :

$$
\vec{v}_{A}^{\beta}(z \mid \vec{k})=k^{2}\left(\delta_{\alpha \beta}-\frac{k_{\alpha} k_{\beta}}{k^{2}}\right) \sin \left(\frac{(2 n+1) \pi}{2 h} z\right) \hat{e}_{\alpha} .
$$

Note that the modes with dispersion relation $\omega=c_{l, t} k$ that occur in an infinite isotropic medium are not normal modes of the plate. The lowest shear mode of the plate has dispersion relation $\omega=c_{t} k$, but it is only polarized in the plane of the plate.

\section{Displacement velocity due to a screw dislocation moving perpendicularly to the surfaces of the plate}

We consider a straight screw dislocation that is parallel to the $z$ axis, or perpendicular to the surfaces of the plate, with a Burgers vector $\vec{b}=b \hat{z}$, in arbitrary motion. The dislocation as a curve is described by the location of its points as a function of time: $\vec{X}(\sigma, \tau)=\left(X_{1}(\tau), X_{2}(\tau), \sigma\right)$, with $\tau$ the time, and $\sigma$ parametrizing the curve. However, the dislocation loop or integration curve $L\left(t^{\prime}\right)$ of Eq. (4) should also include segments on the surfaces of the plate and one at infinity (that it is easily seen not to contribute), in order to close the loop. The segments on the surfaces of the plate do not contribute to the displacement velocity due to the boundary condition satisfied by the Green's function on these surfaces: the indices $p$ and $q$ in Eq. (何) are different from $z$ on the segments on the surface, and so, because of the term $\epsilon_{j p q}, j$ must be equal to $z$, thus $\sigma_{i z}^{G m}\left(x^{\prime}, x ; t-t^{\prime}\right)$ is evaluated at the surfaces $\left(x^{\prime}=x_{S}^{\prime}\right)$ where it vanishes. The expression in Eq. (4) for the displacement velocity is then:

$$
V_{m}(\vec{x}, t)=-b \int_{-\infty}^{\infty} d \tau \int_{-h}^{h} d \sigma C_{z \alpha k l} \epsilon_{\alpha \beta} \frac{\partial X_{\beta}}{\partial \tau} \frac{\partial}{\partial x_{l}^{\prime}} G_{m k}\left(z, z^{\prime} ; \vec{R}-\vec{R}^{\prime} ; t-\tau\right),
$$

with the convention that greek indices take values $(1,2)$ or $(x, y)$, and $\epsilon_{12}=-\epsilon_{21}=1, \epsilon_{11}=\epsilon_{22}=0$. Thus:

$$
\begin{aligned}
V_{m}(\vec{x}, t)= & -b \rho c_{t}^{2} \epsilon_{\alpha \beta} \int_{-\infty}^{\infty} d \tau \frac{\partial X_{\beta}}{\partial \tau} \int_{-h}^{h} d \sigma\left\{-\frac{\partial}{\partial x^{\alpha}} G_{m z}(\vec{x}, \vec{X}(\sigma, \tau) ; t-\tau)\right. \\
& \left.+\frac{\partial}{\partial \sigma} G_{m \alpha}(\vec{x}, \vec{X}(\sigma, \tau) ; t-\tau)\right\}
\end{aligned}
$$


The in-plane Fourier components of the displacement velocity, are defined as:

$$
V_{m}^{\omega}(z \mid \vec{k})=\int_{-\infty}^{\infty} d t \int d \vec{R} e^{-i \omega t+i \vec{k} \cdot \vec{R}} V_{m}(\vec{R}, z, t) .
$$

Replacing this into (18) leads to

$$
V_{m}^{\omega}(z \mid \vec{k})=-\epsilon_{\alpha \beta} I_{\beta}^{\omega}(\vec{k}) F_{m \alpha}^{\omega}(z \mid \vec{k}),
$$

with

$$
I_{\beta}^{\omega}(\vec{k}) \equiv \int_{-\infty}^{\infty} d \tau e^{-i \omega \tau+i \vec{k} \cdot \vec{X}(\tau)} \frac{\partial X_{\beta}}{\partial \tau}(\tau),
$$

a factor that depends on the velocity of the dislocation, and $F_{m \alpha}^{\omega}(z \mid \vec{k})$ a factor that represents the medium's response:

$$
F_{m \alpha}^{\omega}(z \mid \vec{k}) \equiv b \rho c_{t}^{2}\left\{i k_{\alpha} \int_{-h}^{h} d \sigma g_{m z}^{\omega}(z, \sigma \mid \vec{k})+\int_{-h}^{h} d \sigma \frac{\partial}{\partial \sigma} g_{m \alpha}^{\omega}(z, \sigma \mid \vec{k})\right\}
$$

After some algebra, the following expressions for $F_{m \alpha}^{\omega}(z \mid \vec{k})$ can be obtained:

$$
\begin{aligned}
F_{z \alpha}^{\omega}(z \mid \vec{k}) & =\frac{i k_{\alpha} b}{q_{t}^{2}}\left\{1-i \frac{\left(v_{A}^{L}\right)_{z}^{\omega}(z \mid \vec{k})}{\Delta_{a}\left(k^{2}, \omega^{2}\right)}\right\} \\
F_{\delta \alpha}^{\omega}(z \mid \vec{k}) & =\frac{k_{\alpha} b}{q_{t}^{2} \Delta_{a}\left(k^{2}, \omega^{2}\right)}\left(v_{A}^{L}\right)_{\delta}^{\omega}(z \mid \vec{k}) .
\end{aligned}
$$

These factors $F_{m \alpha}^{\omega}(z \mid \vec{k})$ have poles when $\Delta_{a}\left(k^{2}, \omega^{2}\right)=0$, which gives the dispersion relation of the antisymmetric Rayleigh-Lamb modes of the plate. The terms associated with these poles are proportional to the Rayleigh-Lamb mode $\vec{v}_{A}^{L \omega}(z \mid \vec{k})$ introduced in Eq. (12). This shows that the principal contribution (it comes from $\Delta_{a} \rightarrow 0$, or $\omega \sim \omega_{n}^{a}(\vec{k})$ : the dispersion relation of the antisymmetric Rayleigh-Lamb modes) to the displacement velocity field due to a screw dislocation moving perpendicularly to the surfaces of the plate comes from the excitation of the antisymmetric Rayleigh-Lamb modes associated with $\left(\vec{v}_{A}^{L}\right)^{\omega}(z \mid \vec{k})$. When Fourier transforming back to configuration space it becomes clear that only the poles contribute, as they should. An explicitely worked out example is given in Section 7.

\section{Displacement velocity due to an edge dislocation moving perpendicularly to the surfaces of the plate}

We consider a straight edge dislocation parallel to the $z$ axis, or perpendicular to the surfaces of the plate, with a Burgers vector $\vec{b}=b_{x} \hat{x}+b_{y} \hat{y}=b_{\gamma} \hat{e}_{\gamma}$, and moving arbitrarily. According to Eq. (㺼, for this edge dislocation the displacement velocity becomes:

$$
V_{m}(\vec{x}, t)=-\left.b_{\gamma} \epsilon_{\alpha \beta} C_{\gamma \alpha k l} \int_{-\infty}^{\infty} d \tau \int_{-h}^{h} d z^{\prime} \frac{\partial X_{\beta}}{\partial \tau} \frac{\partial}{\partial x_{l}^{\prime}} G_{m k}\left(z, z^{\prime} ; \vec{R}-\vec{R}^{\prime} ; t-\tau\right)\right|_{\vec{R}^{\prime}=\vec{X}(\tau)}
$$

Here again, there is no contribution from the segments of the loop $L\left(t^{\prime}\right)$ that are on the surfaces of the plate and at infinity, for the same reasons that were given for a screw dislocation. Calculating 
the Fourier components of the displacement velocity, one obtains them as the product of two factors:

$$
V_{m}^{\omega}(z \mid \vec{k})=-\epsilon_{\alpha \beta} I_{\beta}^{\omega}(\vec{k}) P_{m \alpha}^{\omega}(z \mid \vec{k}),
$$

where $I_{\beta}^{\omega}(\vec{k})$ is the factor dependent on the dislocation's velocity, Eq. (21), and $P_{m \alpha}^{\omega}(z \mid \vec{k})$ is a factor that represents the medium response:

$$
\begin{aligned}
& P_{z \alpha}^{\omega}(z \mid \vec{k})=\frac{A_{\alpha}\left(\vec{b}, \vec{k}, \omega^{2}\right)}{\Delta_{s}\left(k^{2}, \omega^{2}\right)}\left(v_{S}^{z}\right)_{z}^{\omega}(z \mid \vec{k}) \\
& P_{\gamma \alpha}^{\omega}(z \mid \vec{k})=B_{\gamma \alpha}\left(\vec{b}, \vec{k}, \omega^{2}\right)+\frac{C_{\gamma \alpha}\left(\vec{b}, \vec{k}, \omega^{2}\right)}{q_{t}^{2}}+\frac{A_{\alpha}\left(\vec{b}, \vec{k}, \omega^{2}\right)}{\Delta_{s}\left(k^{2}, \omega^{2}\right)}\left(v_{S}^{z}\right)_{\gamma}^{\omega}(z \mid \vec{k}),
\end{aligned}
$$

with:

$$
\begin{aligned}
A_{\alpha}\left(\vec{b}, \vec{k}, \omega^{2}\right) & \equiv-\frac{\left(\gamma^{2}-2\right)}{q_{l}^{2} \gamma^{2}}\left[\left(\gamma^{2}-2\right) k^{2} b_{\alpha}+2 k_{\alpha} k_{\rho} b_{\rho}\right], \\
B_{\gamma \alpha}\left(\vec{b}, \vec{k}, \omega^{2}\right) & \equiv i \frac{k_{\gamma}}{q_{l}^{2}}\left\{\frac{\left(\gamma^{2}-2\right)}{\gamma^{2}} b_{\alpha}+2 \frac{c_{t}^{2}}{\omega^{2}} k_{\rho} b_{\rho} k_{\alpha}\right\}, \\
C_{\gamma \alpha}\left(\vec{b}, \vec{k}, \omega^{2}\right) & \equiv i\left(k_{\alpha} b_{\gamma}+\delta_{\alpha \gamma} k_{\rho} b_{\rho}-2 \frac{c_{t}^{2}}{\omega^{2}} k_{\rho} b_{\rho} k_{\alpha} k_{\gamma}\right),
\end{aligned}
$$

and $\gamma^{2} \equiv\left(c_{l} / c_{t}\right)^{2}$. Note that the terms of $P_{m \alpha}^{\omega}(z \mid \vec{k})$ which have poles at $\Delta_{s}\left(k^{2}, \omega^{2}\right)=0$ are proportional to the symmetric normal mode of the plate, $\left(\vec{v}_{S}^{z}\right)^{\omega}(z \mid \vec{k})$, of Eq. (14). There is also a pole at $q_{t}^{2}=0$ with non zero residue associated. This shows that the principal contribution (it comes from $\Delta_{s} \rightarrow 0$, or $\omega \sim \omega_{n}^{s}(\vec{k})$ : the dispersion relation of the symmetric Rayleigh-Lamb modes) to the displacement velocity comes from the excitation of the Rayleigh-Lamb mode $\left(\vec{v}_{S}^{z}\right)^{\omega}(z \mid \vec{k})$, and also from the lowest lying shear mode. As in the case of the screw dislocation, going back to configuration space shows, via Cauchy's theorem, that only those modes contribute.

\section{Dynamic stresses on the plate}

Since the elastic stresses satisfy:

$$
\frac{\partial}{\partial t} \sigma_{i j}=C_{i j l m} \frac{\partial V_{m}}{\partial x_{l}}(\vec{x}, t)
$$

for $\omega \neq 0$ its Fourier components are:

$$
\sigma_{i j}^{\omega}(z \mid \vec{k})=\frac{C_{i j l m}}{i \omega} \nabla_{l} V_{m}^{\omega}(z \mid \vec{k}),
$$

with $\nabla_{l} \equiv\left(-i k_{x},-i k_{y}, \partial / \partial z\right)$. We shall check that this expression satisfies the appropriate boundary conditions at the free surfaces.

\subsection{Screw dislocation}

Using Eqs. (20), (33), one obtains the following expression for the stress produced by a dislocation that moves perpendicularly to the surfaces of the plate $(\omega \neq 0)$ :

$$
\sigma_{m z}^{\omega}(z \mid \vec{k})=-\frac{1}{i \omega} I_{\beta}^{\omega}(\vec{k}) \epsilon_{\alpha \beta} \kappa_{m z}^{\alpha \omega}(z \mid \vec{k}),
$$


with:

$$
\begin{aligned}
\kappa_{\alpha \omega}^{z z}(z \mid \vec{k}) & \equiv \rho c_{t}^{2}\left\{-i k_{\gamma}\left(\gamma^{2}-2\right) F_{\gamma \alpha}^{\omega}(z \mid \vec{k})+\gamma^{2} \frac{\partial}{\partial z} F_{z \alpha}^{\omega}(z \mid \vec{k})\right\} \\
\kappa_{\delta z}^{\alpha \omega}(z \mid \vec{k}) & \equiv \rho c_{t}^{2}\left\{-i k_{\delta} F_{z \alpha}^{\omega}(z \mid \vec{k})+\frac{\partial}{\partial z} F_{\delta \alpha}^{\omega}(z \mid \vec{k})\right\}
\end{aligned}
$$

Using the expressions obtained for $F_{m \alpha}^{\omega}(z \mid \vec{k})$ (see Eqs. (23), (24)), one obtains:

$$
\kappa_{\delta z}^{\alpha \omega}(z \mid \vec{k}) \equiv b \rho c_{t}^{2} \frac{k_{\delta} k_{\alpha}}{q_{t}^{2}}\left\{1-\frac{1}{\Delta_{a}}\left[\left(k^{2}+q_{t}^{2}\right)^{2} \sinh \left(q_{l} h\right) \cosh \left(q_{t} z\right)-4 q_{l} q_{t} k^{2} \sinh \left(q_{t} h\right) \cosh \left(q_{l} z\right)\right]\right\} .
$$

If one evaluates the stress $\sigma_{\gamma z}^{\omega}$ on the surfaces of the plate $z= \pm h$, one sees, using Eq. (36), that it vanishes, as it should for a traction free surface. Also,

$$
\kappa_{z z}^{\alpha \omega}(z \mid \vec{k})=b \rho c_{t}^{2} \frac{2 i k_{\alpha} k^{2}\left(k^{2}+q_{t}^{2}\right)}{q_{t} \Delta_{a}}\left\{\sinh \left(q_{t} h\right) \sinh \left(q_{l} z\right)-\sinh \left(q_{l} h\right) \sinh \left(q_{t} z\right)\right\} .
$$

This is also zero on the surfaces of the plate $z= \pm h$, as it should. The other components of the stress also decompose as the product of two factors.

\subsection{Edge dislocation}

The Fourier components of the stress, $\sigma_{m z}^{\omega}(z \mid \vec{k})$, produced by an edge dislocation moving perpendicularly to the surfaces of the plate $(\omega \neq 0)$ are decomposed also into two factors:

$$
\sigma_{m z}^{\omega}(z \mid \vec{k})=-\frac{1}{i \omega} \epsilon_{\alpha \beta} I_{\beta}^{\omega}(\vec{k}) Q_{m z}^{\alpha \omega}(z \mid \vec{k})
$$

with:

$$
\begin{aligned}
Q_{z z}^{\alpha \omega}(z \mid \vec{k})= & D_{\alpha}(\vec{k}, \vec{b})\left\{1-\frac{1}{\Delta_{s}\left(k^{2}, \omega^{2}\right)}\left[\left(k^{2}+q_{t}^{2}\right)^{2} \sinh \left(q_{t} h\right) \cosh \left(q_{l} z\right)\right.\right. \\
& \left.\left.-4 k^{2} q_{l} q_{t} \sinh \left(q_{l} h\right) \cosh \left(q_{t} z\right)\right]\right\}, \\
Q_{\gamma z}^{\alpha \omega}(z \mid \vec{k})= & \frac{2 i k_{\gamma}\left(k^{2}+q_{t}^{2}\right)}{q_{l} \Delta_{s}\left(k^{2}, \omega^{2}\right)} D_{\alpha}(\vec{k}, \vec{b})\left\{\sinh \left(q_{t} h\right) \sinh \left(q_{l} z\right)-\sinh \left(q_{l} h\right) \sinh \left(q_{t} z\right)\right\},
\end{aligned}
$$

and:

$$
D_{\alpha}(\vec{k}, \vec{b}) \equiv \rho c_{t}^{2} \frac{\left(\gamma^{2}-2\right)}{\gamma^{2}}\left[\left(\gamma^{2}-2\right) k^{2} b_{\alpha}+2 k_{\alpha} k_{\rho} b_{\rho}\right] .
$$

These expressions for $Q_{\alpha \omega}^{m z}(z \mid \vec{k})$ assure that the components of the stress $\sigma_{i z}^{\omega}$ are null on the surfaces of the plate.

\section{Example: a uniformly moving screw dislocation}

We consider the case of a screw dislocation that moves perpendicularly to the surfaces of a plate, with uniform velocity $V_{o} \hat{x}$. We will make a parallel with the case of the same dislocation moving 
in an infinite medium, for which the particle velocity is well known [13]. In the case of a thin layer, according to Eqs. (19), (20), the displacement velocity in coordinate space is:

$$
V_{m}(\vec{x}, t)=-\frac{1}{(2 \pi)^{3}} \epsilon_{\alpha \beta} \int d \vec{k} \int d \omega e^{i \omega t-i \vec{k} \cdot \vec{x}} I_{\beta}^{\omega}(\vec{k}) F_{m \alpha}^{\omega}(z \mid \vec{k}) .
$$

For a screw dislocation moving in a plate the factor $F_{m \alpha}^{\omega}(z \mid \vec{k})$ was given in Eqs. (23), (24). In an infinite medium the analogous quantity is:

$$
F_{m \alpha}^{\omega}(z \mid \vec{k})=\frac{i k_{\alpha} b}{q_{t}^{2}} \delta_{m z}
$$

which shows that, in this case, only shear modes polarized in the $z$ direction are excited, since there is a pole at $q_{t}^{2}=0$ or $\omega= \pm c_{t} k$. Notice that in the case of the plate there is an "apparent" pole at $q_{t}^{2}=0$ also, but its residue is zero, meaning that "infinite medium" shear modes are not excited.

Using the definition of $I_{\beta}^{\omega}(\vec{k})$ given in Eq. (21), Eq. (42) for the displacement velocity field component $m=3=z$ in the plate, call it $V_{z}^{P}(\vec{x}, t)$, can be written as (we will concentrate on the component $m=3=z$, which is the only nonvanishing component in the infinite medium case):

$$
V_{z}^{P}(\vec{x}, t)=-\frac{b V_{o} \delta_{\beta x}}{(2 \pi)^{3}} \epsilon_{\alpha \beta} \int d \vec{k} \int d \omega \int d \tau e^{i \omega(t-\tau)-i \vec{k} \cdot\left(\vec{x}-V_{o} \tau \hat{x}\right)} \frac{i k_{\alpha}}{q_{t}^{2}}\left\{1-\frac{i\left(v_{A}^{L}\right)_{z}^{\omega}(z \mid \vec{k})}{\Delta_{a}\left(k^{2}, \omega^{2}\right)}\right\}
$$

The integrand in this expression has poles at $\Delta_{a}\left(k^{2}, \omega^{2}\right)=0$. These poles give the dispersion relation of the antisymmetric Rayleigh-Lamb modes of the plate, $\omega=\omega_{n}^{a}(k)+i \epsilon$. A small positive imaginary part has been added in order to satisfy causality: if $\tau>t$ there are no contributions because one closes the contour of the $\omega$ integral in the lower half plane, where there are no poles. When $t>\tau$ we do the integral over $\omega$ by closing the contour on the upper half plane, and one obtains the contribution from all the poles already mentioned:

$$
V_{z}^{P}(\vec{x}, t)=\left.\frac{b V_{o}}{(2 \pi)^{2}} \epsilon_{\alpha x} \frac{\partial}{\partial x_{\alpha}} \sum_{n} \int d \vec{k} \int_{-\infty}^{t} d \tau e^{i \omega_{n}(k)(t-\tau)-i \vec{k} \cdot\left(\vec{x}-V_{o} \tau \hat{x}\right)} \frac{\left(v_{A}^{L}\right)_{z}^{\omega}(z \mid \vec{k})}{q_{t}^{2} \frac{\partial \Delta_{a}}{\partial \omega}\left(k^{2}, \omega^{2}\right)}\right|_{\omega=\omega_{n}(k)}
$$

while the analogous expression in the infinite medium case for the $z$ component of the displacement velocity field, $V_{z}^{\infty}(\vec{x}, t)$, comes from calculating the residues at the poles $\omega_{n}= \pm c_{t} k$. This gives:

$$
V_{z}^{\infty}(\vec{x}, t)=-\frac{i b V_{o} c_{t}^{2} \epsilon_{\alpha x}}{(2 \pi)^{2}} \frac{\partial}{\partial x_{\alpha}} \int d \vec{k} \int_{-\infty}^{t} d \tau e^{-i \vec{k} \cdot\left(\vec{x}-V_{o} \tau \hat{x}\right)}\left\{\frac{e^{i k c_{t}(t-\tau)}}{2 k c_{t}}-\frac{e^{-i k c_{t}(t-\tau)}}{2 k c_{t}}\right\} .
$$

The last two equations show that the solution for $V_{z}(\vec{x}, t)$ can be written as a superposition of contributions from different modes. The integration over $\tau$ can be done, and it renders for the case of the plate:

$$
V_{z}^{P}(\vec{x}, t)=-\left.\frac{i b V_{o}}{(2 \pi)^{2}} \frac{\partial}{\partial y} \sum_{n} \int d \vec{k} \frac{e^{-i \vec{k} \cdot\left(\vec{x}-V_{o} t \hat{x}\right)}}{\left(\omega_{n}(k)-k_{x} V_{o}\right)} \frac{\left(v_{A}^{L}\right)_{z}^{\omega}(z \mid \vec{k})}{q_{t}^{2} \frac{\partial}{\partial \omega} \Delta_{a}\left(k^{2}, \omega^{2}\right)}\right|_{\omega=\omega_{n}(k)}
$$

and in the case of the infinite medium:

$$
V_{z}^{\infty}(\vec{x}, t)=-\frac{b V_{o} c_{t}}{2(2 \pi)^{2}} \frac{\partial}{\partial y} \int \frac{d \vec{k}}{k} e^{-i \vec{k} \cdot\left(\vec{x}-V_{o} t \hat{x}\right)}\left\{\frac{1}{\left(k c_{t}-k_{x} V_{o}\right)}-\frac{1}{\left(-k c_{t}-k_{x} V_{o}\right)}\right\} .
$$


These last two equations show that there would be a large contribution to the final result from the region where the "Doppler shifted" frequency of the modes $\left(\omega_{n}(k)-k_{x} V_{o}\right)$ is approximately zero (if that is possible for a given $V_{o}$ ). For example, in the infinite medium case if the velocity $V_{o}$ of the dislocation goes beyond $c_{t}$, one gets Cerenkov type radiation. But, for the case of the plate, the phase velocities $\omega_{n}(k) / k_{x}$ are always greater than $V_{R}$, the Rayleigh wave velocity, meaning that there is no large excitation of modes at uniform velocities $V_{o}<V_{R}$.

The angular integral over $\vec{k}$ can't be done analytically for the case of the plate. We continue with the infinite medium case in order to illustrate that one can get the exact solution as a superposition over the shear modes excited. If $\vec{k}=k(\cos \theta, \sin \theta)$, then Eq. (48) can be written:

$$
V_{z}(\vec{x}, t)=\frac{i b c_{t}}{2(2 \pi)^{2}} \int_{0}^{\infty} d k \int_{-\pi}^{\pi} d \theta \sin \theta e^{-i k\left(x-V_{o} t\right) \cos \theta-i k y \sin \theta}\left\{\frac{1}{\left(\frac{c_{t}}{V_{o}}-\cos \theta\right)}+\frac{1}{\left(\frac{c_{t}}{V_{o}}+\cos \theta\right)}\right\} .
$$

Doing the integral over $k$ :

$$
V_{z}(\vec{x}, t)=\frac{b c_{t}}{2(2 \pi)^{2}} \int_{-\pi}^{\pi} d \theta \frac{\sin \theta}{\left(\left(x-V_{o} t\right) \cos \theta+y \sin \theta\right)}\left\{\frac{1}{\left(\frac{c_{t}}{V_{o}}-\cos \theta\right)}+\frac{1}{\left(\frac{c_{t}}{V_{o}}+\cos \theta\right)}\right\} .
$$

This angular integral can be done by using the change of variables $z=e^{i \theta}$, then it becomes an integral over the unit circle in the complex plane, and then one applies Cauchy's theorem. The result is:

$$
V_{z}(\vec{x}, t)=\frac{b V_{o}}{2 \pi} \frac{y \sqrt{1-\left(\frac{V_{o}}{c_{t}}\right)^{2}}}{\left[\left(x-V_{o} t\right)^{2}+y^{2}\left(1-\left(\frac{V_{o}}{c_{t}}\right)^{2}\right)\right]},
$$

which corresponds to the exact solution that can be obtained in a simpler way [13]:

$$
U_{z}(\vec{x}, t)=\frac{b}{2 \pi} \tan ^{-1}\left(\frac{y \sqrt{1-\left(\frac{V_{o}}{c_{t}}\right)^{2}}}{\left(x-V_{o} t\right)}\right) .
$$

This is, of course, the static solution for a screw dislocation in an infinite medium, $U_{z}=(b / 2 \pi) \phi=$ $(b / 2 \pi) \tan ^{-1}(y / x)$, when Lorentz-transformed to a frame moving with velocity $V_{0}$.

\section{Relation with experiments on fast fracture}

Experiments on fast fracture of glass and plexiglass [1, 2, 3, 4, 5, 6] have been made in mode I loading, which means that, in order to use our results, the crack should be modeled by edge dislocations. Our results about the motion of edge dislocations show that the mode excited in this case is the Rayleigh-Lamb mode associated with $\left(\vec{v}_{S}^{z}\right)^{\omega}(z \mid \vec{k})$. Moreover, experiments exhibit acoustic and surface roughness spectra that have strong peaks at frequencies corresponding to the lowest lying long wavelength Rayleigh-Lamb modes [7], and it is tempting to think that long wavelength modes, requiring less energy, will be more efficiently excited. The long wavelength solution for the dispersion relation of these modes comes from taking the limit $k \rightarrow 0$ in $\Delta_{s}\left(k^{2}, \omega^{2}\right)=0$, and leads to two possibilities: $\cos \left(\omega h / c_{l}\right)=0$ or "odd" frequencies $\omega_{n}=(2 n+1) \pi c_{l} / 2 h(n=1,2, \ldots)$ and $\sin \left(\omega h / c_{t}\right)=0$ or "even" frequencies $\omega_{m}=m \pi c_{t} / h(m=1,2, \ldots)$. The limit $k \rightarrow 0$ for the mode $\vec{v}_{S}^{z}(z \mid \vec{k})$ is:

$$
\vec{v}_{S}^{z}(z \mid \vec{k}) \simeq-i \frac{\omega^{3}}{c_{l} c_{t}^{2}} \sin \left(\frac{\omega}{c_{t}} h\right) \sin \left(\frac{\omega}{c_{l}} z\right) \hat{z}
$$


This expression goes to zero for the frequencies previously called "even", which means that the amplitude of excitation of these modes will be lower than the corresponding amplitudes associated with the "odd" frequencies. The fact that experiments deal with a crack, that is with many dislocations side by side, rather than a single dislocation will not change this fact and it may well happen that deviations from straight-front crack tip behaviour must be considered.

\section{Concluding remarks}

In this paper we have given an expression for the elastodynamic fields that are excited in a thin plate by a dislocation, perpendicular to the faces of the plate, in arbitray motion. This expression is a convolution of the impulse response of the plate with a source function that is localized at the dislocation position. An explicit expression for the impulse response has been determined in Fourier space as a rational function, with poles at the well known Rayleigh Lamb modes, both for screw and edge dislocations. Application to the case of a uniformly moving screw shows that large amounts of radiation are to be expected when the dislocation's velocity coincides with the phase velocity of one of the modes.

This work is part of an ongoing project that attempts to understand recently observed dynamic crack instabilities in thin plates in terms of the interaction of the crack tip with the wave modes of the plate. We hope the formalism presented in this work will prove helpful in advancing this project.

\section{Acknowledgments}

We thank J. F. Boudet and S. Ciliberto for communicating their experimental results previous to publication. This work was supported in part by Fondecyt Grants 3950011 and 1960892, Fundación Andes, and by a Cátedra Presidencial en Ciencias.

\section{References}

[1] S. Gross, J. Fineberg, M. Marder, W. McCormick, H. Swinney, "Acoustic emissions from rapidly moving cracks", Phys. Rev. Lett. 71, 3162 (1993).

[2] E. Sharon, S.P. Gross and J. Fineberg, "Local crack branching as a mechanism for instability in dynamic fracture", Phys. Rev. Lett. 74, 5096 (1995).

[3] E. Sharon, S.P. Gross and J. Fineberg, "Energy dissipation in dynamic fracture", Phys. Rev. Lett. 76, 2117 (1996).

[4] E. Sharon and J. Fineberg, "Microbranching instability and the dynamic fracture of brittle materials", Phys. Rev. B 54, 7128 (1996).

[5] J.F. Boudet, S. Ciliberto and V. Steinberg, "Experimental study of the instability of crack propagation in brittle materials", Europhys. Lett. 30, 337 (1995).

[6] J.F. Boudet, S. Ciliberto and V. Steinberg, "Dynamics of crack propagation in brittle materials", J. Phys. II France 6 , 1493 (1996). 
[7] F. Lund, "Elastic forces that do no work and the dynamics of fast cracks", Phys. Rev. Lett. $76,2742(1996)$.

[8] F. Lund, "Response of a stringlike dislocation loop to an external stress", J. Mater. Res. 3, 280 (1988).

[9] J. D. Achenbach, Wave Propagation in Elastic Solids, North-Holland, Amsterdam (1984).

[10] T. Mura, "Continuous distribution of moving dislocations", Philos. Mag. 8, 843 (1963).

[11] R. Arias and F. Lund, "Elastic fields of stationary and moving dislocations in three dimensional finite samples", submitted to J. Mech. Phys. Solids (November 1997), cond-mat/9711155.

[12] K.A. Chishko, "Dynamical Green's tensor and elastic fields of a system of moving dislocation loops in an isotropic plate", Sov. Phys. Acoust. 35, 307 (1989).

[13] J.P. Hirth and J. Lothe, Theory of dislocations, Wiley, New York (1982).

\section{Appendix: Fourier components of the plate's Green's func- tion}

In this Appendix we reproduce the expression for the Green's function of a thin plate given by Chishko [12]. According to Eq. (5) the Fourier components of the plate's Green's function are:

$$
g_{i m}^{\omega}\left(z, z^{\prime} \mid \vec{k}\right)=g_{i m}^{\infty \omega}\left(z-z^{\prime} \mid \vec{k}\right)-h_{i m}^{\omega}\left(z, z^{\prime} \mid \vec{k}\right) .
$$

From Eq. (6):

$$
\begin{aligned}
h_{i m}^{\omega}\left(z, z^{\prime} \mid \vec{k}\right) & =\sum_{a}( \pm)\left[-i k_{\beta} C_{n z \beta p} g_{i n}^{P \omega}\left(z, \xi^{a} \mid \vec{k}\right) g_{p m}^{\infty \omega}\left(\xi^{a}-z^{\prime} \mid \vec{k}\right)\right. \\
& \left.+C_{n z z p} g_{i n}^{P \omega}\left(z, \xi^{a} \mid \vec{k}\right) \frac{\partial}{\partial z} g_{p m}^{\infty \omega}\left(\xi^{a}-z^{\prime} \mid \vec{k}\right)\right],
\end{aligned}
$$

where $( \pm)$ refers to $a=1,2$ or $\xi^{a}= \pm h$. The Fourier transforms of the infinite medium's Green's function are [12]:

$$
g_{i j}^{\infty \omega}\left(z-z^{\prime} \mid \vec{k}\right)=-\frac{1}{2 \rho \omega^{2}}\left\{s_{i}^{l} s_{j}^{l} \frac{e^{-q_{l}\left|z-z^{\prime}\right|}}{q_{l}}-\left[s_{i}^{t} s_{j}^{t}+\frac{\omega^{2}}{c_{t}^{2}} \delta_{i j}\right] \frac{e^{-q_{t}\left|z-z^{\prime}\right|}}{q_{t}}\right\},
$$

with $\vec{s}^{l, t} \equiv\left(i k_{x}, i k_{y}, q_{l, t} s\left(z-z^{\prime}\right)\right), s(x) \equiv \operatorname{sign}(x)$, and $q_{l, t} \equiv \sqrt{k^{2}-\omega^{2} / c_{l, t}^{2}}$. The Fourier transforms of the Green's function $G_{i j}^{P}$ (see Eqs. (7), (8)) are [12]:

$$
g_{i j}^{P \omega}(z, \xi \mid \vec{k})=\frac{1}{2 \rho c_{t}^{2}} \sum_{\lambda=l, t} M_{i j}^{\lambda \omega}(z, \xi \mid \vec{k}) .
$$

Here $M_{i j}^{\lambda \omega}(z, \xi \mid \vec{k})$ are matrices with components:

$$
\begin{array}{rll}
M_{z z}^{l \omega}=q_{l}\left(k^{2}+q_{t}^{2}\right) \Phi_{2}(z \mid \xi) & ; & M_{z z}^{t \omega}=-2 q_{l} k^{2} \Phi_{2}(\xi \mid z) \\
M_{z \beta}^{l \omega}=-2 i q_{l} q_{t} k_{\beta} \Phi_{4}(z \mid \xi) & ; & M_{z \beta}^{t \omega}=i\left(k^{2}+q_{t}^{2}\right) k_{\beta} \Phi_{3}(\xi \mid z) \\
M_{\alpha z}^{l \omega}=-i\left(k^{2}+q_{t}^{2}\right) k_{\alpha} \Phi_{3}(z \mid \xi) & ; \quad & M_{\alpha z}^{t \omega}=2 i q_{l} q_{t} k_{\alpha} \Phi_{4}(\xi \mid z) \\
M_{\alpha \beta}^{l \omega}=-2 q_{t} k_{\alpha} k_{\beta} \Phi_{1}(z \mid \xi) & ; &
\end{array}
$$




$$
M_{\alpha \beta}^{t \omega}=\frac{k_{\alpha} k_{\beta}}{k^{2}} q_{t}\left(k^{2}+q_{t}^{2}\right) \Phi_{1}(\xi \mid z)+\left(\delta_{\alpha \beta}-\frac{k_{\alpha} k_{\beta}}{k^{2}}\right)\left[\frac{\left(k^{2}+q_{t}^{2}\right)^{2}}{q_{t}} \Phi_{1}(\xi \mid z)-4 q_{l} k^{2} \Psi(\xi \mid z)\right]
$$

where $\xi$ is equal to either $h$ or $-h$. The functions $\Phi_{p}(u \mid v)$ and $\Psi(u \mid v)$ are defined as:

$$
\begin{aligned}
\Phi_{1}(u \mid v)= & \frac{1}{\Delta_{s}} \cosh \left(q_{l} u\right) \cosh \left(q_{t} v\right)+\frac{1}{\Delta_{a}} \sinh \left(q_{l} u\right) \sinh \left(q_{t} v\right) \\
\Phi_{2}(u \mid v)= & \frac{1}{\Delta_{s}} \sinh \left(q_{l} u\right) \sinh \left(q_{t} v\right)+\frac{1}{\Delta_{a}} \cosh \left(q_{l} u\right) \cosh \left(q_{t} v\right) \\
\Phi_{3}(u \mid v)= & \frac{1}{\Delta_{s}} \cosh \left(q_{l} u\right) \sinh \left(q_{t} v\right)+\frac{1}{\Delta_{a}} \sinh \left(q_{l} u\right) \cosh \left(q_{t} v\right) \\
\Phi_{4}(u \mid v)= & \frac{1}{\Delta_{s}} \sinh \left(q_{l} u\right) \cosh \left(q_{t} v\right)+\frac{1}{\Delta_{a}} \cosh \left(q_{l} u\right) \sinh \left(q_{t} v\right) \\
\Psi(u \mid v)= & \frac{1}{\Delta_{s}} \operatorname{coth}\left(q_{t} u\right) \sinh \left(q_{l} u\right) \cosh \left(q_{t} v\right) \\
& +\frac{1}{\Delta_{a}} \tanh \left(q_{t} u\right) \cosh \left(q_{l} u\right) \sinh \left(q_{t} v\right),
\end{aligned}
$$

with:

$$
\begin{aligned}
& \Delta_{s}\left(k^{2}, \omega^{2}\right)=\left(k^{2}+q_{t}^{2}\right)^{2} \cosh \left(q_{l} h\right) \sinh \left(q_{t} h\right)-4 k^{2} q_{l} q_{t} \cosh \left(q_{t} h\right) \sinh \left(q_{l} h\right) \\
& \Delta_{a}\left(k^{2}, \omega^{2}\right)=\left(k^{2}+q_{t}^{2}\right)^{2} \sinh \left(q_{l} h\right) \cosh \left(q_{t} h\right)-4 k^{2} q_{l} q_{t} \sinh \left(q_{t} h\right) \cosh \left(q_{l} h\right)
\end{aligned}
$$

